\title{
Bacterial Etiology and Pneumococcal Serotypes in Turkish Children with Acute Otitis Media
}

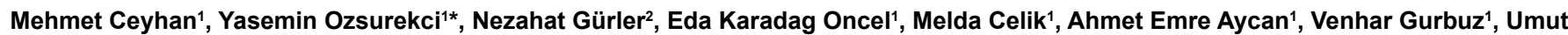
Akyol $^{3}$, Ozan Altuntaş ${ }^{3}$, Adnan Karabent ${ }^{4}$, Yıldız Camcıoğlu ${ }^{5}$, Mehmet Ada $^{6}$, Emre Alhan ${ }^{7}$ and Özlem Özgür ${ }^{7}$

${ }^{1}$ Hacettepe University Faculty of Medicine, Pediatric Infectious Diseases, Ankara, Turkey

${ }^{2}$ Istanbul University Faculty of Medicine, Department of Clinical Microbiology, Istanbul, Turkey

${ }^{3}$ Hacettepe University Faculty of Medicine, Department of Otorhinolaryngology, Ankara, Turkey

${ }^{4}$ Cankırı State Hospital, Cankırı, Turkey

5/stanbul University Faculty of Medicine, Pediatric Infectious Diseases, Istanbul, Turkey

${ }^{6}$ Istanbul University Faculty of Medicine, Department of Otorhinolaryngology, Istanbul, Turkey

${ }^{7}$ Cukurova University Faculty of Medicine, Pediatric Infectious Diseases, Adana, Turkey

\begin{abstract}
Background: Acute otitis media (AOM) is one of the most common childhood diseases requiring antimicrobial prescription drugs in pre-school children. In this study, we sought to describe the bacterial etiology of pediatric cases of AOM in Turkey.

Materials and Methods: This prospective, multi-center, tympanocentesis-based epidemiological study was performed during 2010-2012in children aged between 3 months and 6 years. All isolates were cultured and sero grouped by the Quellung reaction.

Results: During 2010-2012, 38 cases fulfilled the study inclusion criteria. Overall, $45 \%$ of samples were culture positive for bacterial pathogens Streptococcus pneumonia (13.1\%) was the leading cause of bacterial AOM followed by Streptococcus pyogenes $(10.5 \%)$ and $H$. influenzae $(7.9 \%)$. Serotype- 3 was detected in two of the samples, and serotypes 9V, 19, and 19A were isolated from one patient each. S. pneumoniae was detected in $36 \%(4 / 11)$ of otorrhea samples. All $H$. influenzae-positive samples were collected by tympanocentesis. All $H$. influenzae isolates were identified as non-typeable. The pneumococcal serotype coverage rates for PCV-7, PVC-10 and PCV-13 were $20 \%(1 / 5), 20 \%(1 / 5)$, and $80 \%(4 / 5)$, respectively. PHiD-CV (PCV-7 types plus 1, 5, and 7F) targets non-typeable $H$. influenzae, and 4 of $38(11 \%)$ of the pathogens causing episodes of AOM were also covered.

Conclusion and Recommendation: In Turkey, S.pneumonia remains the most common pathogen in children with AOM. Both S. pneumonia and non-typeable $\mathrm{H}$. influenzae represent important targets for vaccination strategies to reduce AOM in children. Based on our results, conjugated pneumococcal vaccines may have potential impact to decrease the burden of AOM.
\end{abstract}

Keywords: Acute otitis media; Streptococcus pneumoniae; Children; Vaccine

\section{Introduction}

Acute otitis media (AOM) is one of the most commonly diagnosed childhood infections, and this is true not only in the USA where it accounts for more than 20 million visits to pediatricians every year. The disease is most prevalent in children younger than 2 years of age [1-3]. The etiology of AOM varies with age, the most frequently implicated agents being viruses such as rhinoviruses, influenza viruses, or respiratory syncytial viruses and bacteria, such as non-encapsulated Haemophilus influenzae, Streptococcus pneumoniae, and Moraxella catarrhalis [4]. Streptococcus pneumoniae and Haemophilus influenzae have been consistently reported to be the two major bacterial pathogens responsible for AOM, mainly by studies in the USA and European countries [5-7]. Three pneumococcal conjugate vaccines (PCVs) are currently licensed for use in children and they have showed modest efficacy against AOM overall [8]. Randomized clinical trials with a 7 -valent pneumococcal conjugate vaccine (PCV-7) in the USA and Finland showed reductions in the incidence of AOM of 6-9\% [9-12]. Another pneumococcal vaccine that has been used the outer membrane protein D carrier derived from $H$. influenzae as a carrier showed 35\% efficacy against clinical AOM, with statistically significant protection against both $S$. pneumoniae and $H$. influenza-related AOM [3]. In children, PCV-13 provides serotype coverage of $90.2 \%$ for AOM [13].
Understanding the pathogens involved in the epidemiology of AOM is important not only for treatment options but also because pneumococcal conjugate vaccines have shown promise in preventing the disease. The potential impact of the vaccines cannot be estimated without comprehensive data on AOM incidence and etiology in the specific area. Because the incidence of the bacteria and serotype distribution of etiologic agents differ from a region to others. However, data on bacterial pathogens causing AOM in Turkey are limited. In this study, we aimed to characterize the bacterial etiology, and serotypes of S. pneumoniae AOM cases in Turkey.

*Corresponding author: Yasemin Ozsurekci, Hacettepe University Medical Faculty, Pediatric Infectious Diseases Unit, Sıhhıye, Ankara 06100, Turkey, Tel: +090 312 3051166; Fax: +090 312 3108241; E-mail: yas.oguz99@yahoo.com, yasemin.ozsurekci@gmail.com

Received December 07, 2013; Accepted December 24, 2013; Published December 28, 2013

Citation: Ceyhan M, Ozsurekci Y, Gürler N, Oncel EK, Celik M, et al. (2013) Bacterial Etiology and Pneumococcal Serotypes in Turkish Children with Acute Otitis Media. J Vaccines Vaccin 5: 214. doi: 10.4172/2157-7560.1000214

Copyright: (c) 2013 Ceyhan M, et al. This is an open-access article distributed under the terms of the Creative Commons Attribution License, which permits unrestricted use, distribution, and reproduction in any medium, provided the original author and source are credited. 


\section{Materials and Methods}

\section{Study population and definitions}

This was a prospective, multi-center, epidemiological study conducted within a routine clinical setting in five pediatric centers in Turkey. Turkish children between 3 and 59 months of age with otitis media from whom a middle ear fluid (MEF) sample was obtained with an indication for tympanocentesis by a near, nose, and throat (ENT) specialist or whom with otorrhea were included. Demographics, medical history, and general symptoms were collected at baseline, and a clinical examination was performed. Children were excluded if they suffered from a severe underlying disease. This study mainly included recurrent or treatment failure AOM. Recurrent AOM was defined as at least three episodes in the past 6 months or at least four episodes in the past 12 months. Patients identified for recruitment were subjects with a new episode of AOM (within $<72 \mathrm{~h}$ of onset) who had not yet received antibiotics for the episode, and subjects who had a diagnosis of AOM within 48-72 h prior to study enrollment and who received antibiotic therapy from a physician but remained symptomatic at the time of study entry (treatment failures). To be considered a new episode, there had to be a symptom-free interval of at least 30 days since resolution of the previous episode. AOM was diagnosed after otoscopic examination of the ear and tympanic membrane by a pediatrician, and was classified according to the otoscopy score (eight grades) (OS-8), which measures the severity of tympanic-membrane inflammation. The diagnosis was then verified upon referral to and examination by an ENT specialist.

\section{Middle ear fluid samples}

A MEF sample was collected by tympanocentesis by needle insertion or otorrhea sample was obtained in the perforated tympanic membrane. In the case of a perforated tympanic membrane, the sample was cultured if it was taken within $24 \mathrm{~h}$ of the perforation. Middle ear fluid was collected by an ENT specialist by tympanocentesis or by sampling of otorrhea after confirmation of AOM. Children were excluded from the analysis if their tympanic membranes had been perforated for more than $48 \mathrm{~h}$.

\section{Microbiology and serotypes}

Because the pathogen distribution of tympanocentesis isolates can be different than otorrhea isolates [14], number of otorrhea samples was limited up to $30 \%$ of total subjects. Samples were analyzed at Microbiology Laboratory of Istanbul University, Faculty of Medicine to isolate bacterial pathogens and determine the serotypes of $S$. pneumoniae. Serotyping was performed by the Quelluing reaction using serotype-specific antisera (Statens Seruminstitut, Copenhagen, Denmark).

Informed consent was obtained from each parent/guardian prior to performall study-specific procedures. The study was reviewed and approved by the Hacettepe University Institutional Ethical Committee (Approval munber: EPI-STREP-111339).

\section{Results}

Between December 2010 and January 2012, 38 patients were eligible for inclusion criteria. Eleven of the 38 (29\%) episodes were classified as treatment failures, and the remaining $27(71 \%)$ of episodes were classified as recurrent cases. All of the AOM episodes were unilateral infections for which a single sample was collected. Of the 38 samples, 27 (71\%) were collected by tympanocentesis, and $11(29 \%)$ were collected by otorrhea.
The age of participants vary from 6 months to 5.8 years in all AOM cases. Twenty three of children were male, and 15 were female. In total, 13 patients (34\%) had received at least one dose of the PCV-7, and only one patient had received at least one dose of influenza vaccine. Antibiotic use within the past 1month was reported for 13 (34\%) of the cases. Irritability was the most frequently reported symptom, followed by ear pain, and tugging.

Overall, 45\% (17/38) of the samples from the 38 episodes yielded cultures with one of the bacteria considered potential pathogens (S. pneumoniae, S. aureus, S. pyogenes, $H$. influenzae or $\alpha$-hemolytic Streptococcus). The most commonly recovered bacteria were $S$. pneumoniae, which were detected in $13.2 \%$ (5/38) of samples, followed by $S$. pyogenes and $H$. influenzae, which were detected in $10.5 \%(4 / 38)$, and $7.9 \%(3 / 38)$, respectively (Table 1$)$. The pneumococcal serotypes isolated from the $S$. pneumoniae positive samples were $3(2 / 5), 9 \mathrm{~V}$ (1/5), 19A (1/5), and 19 (1/5). S. pneumoniae serotype-3 was isolated from one of the patients who were vaccinated with PCV-7. The remaining pneumococcal serotypes were isolated from unvaccinated patients. S. pneumoniae was detected in $36 \%$ (4/11) of samples collected by otorrhea. All of the samples where $H$. influenzae was detected were collected by tympanocentesis (Figure 1). All $H$. influenzae isolates were identified as non-typeable.

\section{Discussion}

Acute otitis media (AOM) is one of the most frequent bacterial infections in children and the primary reason for the prescription of antibiotics by pediatricians $[15,16]$. S. pneumoniae is a major etiologic agent causing various infectious diseases ranging from non-invasive diseases such as AOM, rhinosinusitis and pneumonia to invasive disease such as sepsis and meningitis. Children, especially those below

${ }^{*}$ :patient number, \% percent

Table 1: Etiology of bacteria identified from samples cultured from middle ear fluid of children experiencing episodes of AOM.

\begin{tabular}{|l|c|}
\hline Bacteria & $\mathbf{n}(\%)^{*}$ \\
\hline S. pneumoniae & $5(13.2)$ \\
\hline S. pyogenes & $4(10.5)$ \\
\hline H. influenzae (non-typable) & $3(7.9)$ \\
\hline a-hemolytic streptococcus & $2(5.3)$ \\
\hline Staf. aureus & $1(2.6)$ \\
\hline Staf. auricularis & $1(2.6)$ \\
\hline Pseud. auriginosa & $1(2.6)$ \\
\hline The total of bacteria positive samples & $17(44.7)$ \\
\hline Total & $38(100)$ \\
\hline
\end{tabular}

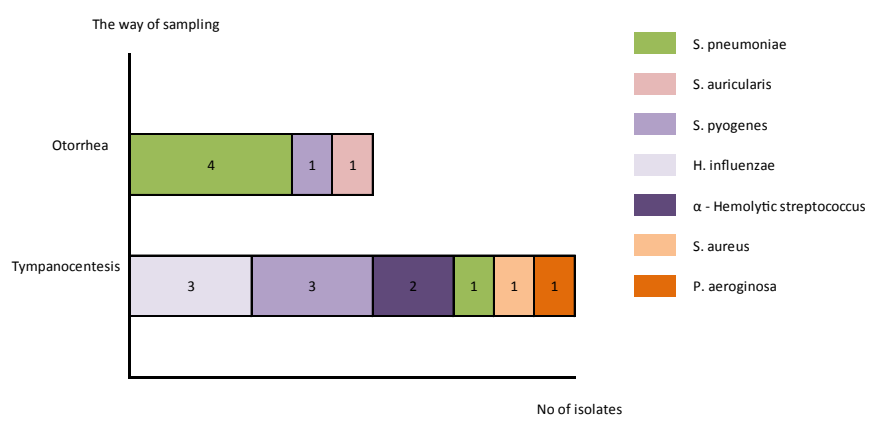

Figure 1: Etiology of bacteria identified from samples that based on the way of sampling. 
the age of 5 , are particularly at risk from invasive infections $[17,18]$. Streptococcus pneumoniae is the most commonly reported bacterial cause of acute otitis media, accounting for $28-55 \%$ of cases $[19,20]$. In this study, $45 \%(17 / 38)$ of samples cultured positive for one of the pathogens under study, which was lower than expected, perhaps due to high antibiotic use before sampling which has been reported as 53 to $58 \%$ [21,22]. S. pneumoniae was the leading cause of bacterial AOM in this study, consistent with other reports from Turkey [23,24], followed by $S$. pyogenes (4/38) and Haemophilus influenzae (3/38). $S$. pneumoniae and $H$. influenzae were the most prevalent species in pediatric cases with AOM in the literature [25,26], as consistently our findings. A higher proportion of $S$. pyogenes was observed in this study compared with others.

Following licensing of PCV-7 in 2000 for use in several countries worldwide, a decrease in invasive pneumococcal disease (IPD), pneumonia, and otitis media of any etiology was observed $[9,10]$. Recent reports from countries where PCV-7 has been implemented into national immunization program (NIP) have shown a minor to moderate decline in the incidence of otitis media visits and antimicrobial consumption to treat this condition [27,28], an increase in non-vaccine S. pneumoniae serotypes, especially of serotype 19A [25], and a proportional increase in the number of $H$. influenzae cases [29] as seen in the present study. Marta Alonso et al. [25] reported that most prevalent pneumococcal serotypes in pediatric cases with AOM were 3 and 19A. Reijtman et al. [26] reported that frequent pneumococcal serotypes were 19A, 9V, and 3 in AOM. Serotypes 3, $9 \mathrm{~V}$ and $19 \mathrm{~A}$ were detected in the present study as consistently with those studies. PCV-7 was implemented in the NIP in 2008 in Turkey, and it was then replaced with 13-valent vaccine (PCV-13) in 2011. Of the children enrolled in the study, $34 \%$ had been vaccinated with PCV-7, whereas none had been vaccinated with PCV-13 because of unavailability in the study period.

Recent studies have introduced two conjugate vaccines: PCV-10 and PCV-13. Data suggested that after changing from PCV-7 to PCV10 , the proportion of serotypes covered would increase to varying degrees in USA, Europe, Africa, and Asia. Changing from PCV-10 to PCV-13 would further improve the coverage of serotypes by $4 \%$ to $7 \%$ globally [30]. Too little information about the incidence and serotype distribution of $S$. pneumoniae in IPD or otitis media during the prepneumococcal conjugate vaccine era in Turkey is available. Based on limited data about serotype distribution, S. pneumoniae seems to be the most common etiologic AOM agent in Turkey [24,31]. Based on the pathogen distribution seen in this study, pneumococcal conjugate vaccines could be an important tool in reducing the burden of AOM in this setting. The pneumococcal serotypes targeted by PCV7 and PCV-13 comprise $20 \%(1 / 5)$ and $80 \%(4 / 5)$, respectively of the $S$. pneumoniae-positive AOM episodes in this study, and coverage was 3\% $(1 / 38)$ for PCV-7 and $11 \%$ (4/38) for PCV-13, respectively, in all AOM episodes. The pneumococcal serotypes targeted by PHiD-CV (PCV-7 types plus 1,5 , and $7 \mathrm{~F})$ comprised $20 \%(1 / 5)$ of the $S$. pneumoniaepositive AOM episodes in this study. Additionally, PHiD-CV targets $H$. influenzae, and thus $11 \%(4 / 38)$ of the pathogens causing episodes of AOM in this study.

S. pneumoniae was found among otorrhea samples in this study, consistent with some other studies, where S. pneumoniae was reported to be more often isolated from otorrhea samples [32,33]. In contrast to Parra et al. [16], in this study, all of $H$. influenzae samples were found in tympanocentesis samples.
Moraxella catarrhalis could not be found in any AOM episode in this study. This finding was not inconsistent with other studies that have reported low percentages of Moraxella [7,16].

This study has some limitations. First, it was performed in a specific setting, and only a relatively small number of children who were admitted to hospital were enrolled. All patients who had acute AOM episodes were excluded. The AOM cases in this study may not accurately represent all AOM cases in Turkey due to potential differences between the enrolled and screened patients. This prevents further generalization of the findings. There may also have bias in the selection of patients. Second, the number of children was small. Thus, there may also be an assessment bias that may affect the reliability of the data.

In conclusion, the bacteriology of otitis media has been studied in several parts of the world; however, there are few current data from Turkey. This assessment of AOM etiology in Turkish children aged 3 months to less than 6 years, who visited pediatric clinics for AOM, showed that $47 \%(8 / 17)$ of all culture-positive samples were positive for S. pneumoniae or H. influenzae. Both S. pneumoniae and non-typeable $H$. influenzae represent important targets for vaccination strategies to reduce AOM in Turkish children. A continuous surveillance program is needed to detect bacteriological and/or serotype modifications that may occur over time and following vaccine interventions.

\section{References}

1. Schappert SM (1992) Office visits for otitis media: United States, 1975-90. Adv Data : 1-19.

2. Advisory Committee on Immunization Practices (2000) Preventing pneumococcal disease among infants and young children. Recommendations of the Advisory Committee on Immunization Practices (ACIP). MMWR Recomm Rep 49: 1-35.

3. Prymula R, Peeters P, Chrobok V, Kriz P, Novakova E, et al. (2006) Pneumococcal capsular polysaccharides conjugated to protein $D$ for prevention of acute otitis media caused by both Streptococcus pneumoniae and nontypable Haemophilus influenzae: a randomised double-blind efficacy study. Lancet 367: 740-748.

4. Fischer T, Singer AJ, Lee C, Thode HC Jr (2007) National trends in emergency department antibiotic prescribing for children with acute otitis media, 1996 2005. Acad Emerg Med 14: 1172-1175

5. Arguedas A, Sher L, Lopez E, Sáez-Llorens X, Hamed K, et al. (2003) Open label, multicenter study of gatifloxacin treatment of recurrent otitis media and acute otitis media treatment failure. Pediatr Infect Dis J 22: 949-956.

6. Casey JR, Pichichero ME (2004) Changes in frequency and pathogens causing acute otitis media in 1995-2003. Pediatr Infect Dis J 23:824-828.

7. Leibovitz E, Jacobs MR, Dagan R (2004) Haemophilus influenzae: a significan pathogen in acute otitis media. Pediatr Infect Dis J 23: 1142-1152.

8. Setchanova LP, Kostyanev T, Alexandrova AB, Mitov IG, Nashev D, et al. (2013) Microbiological characterization of Streptococcus pneumoniae and nontypeable Haemophilus influenzae isolates as primary causes of acute otitis media in Bulgarian children before the introduction of conjugate vaccines. Ann Clin Microbiol Antimicrob 12: 6

9. Black S, Shinefield H, Fireman B, Lewis E, Ray P, et al. (2000) Efficacy, safety and immunogenicity of heptavalent pneumococcal conjugate vaccine in children. Northern California Kaiser Permanente Vaccine Study Center Group. Pediatr Infect Dis J 19: 187-195.

10. Eskola J, Kilpi T, Palmu A, Jokinen J, Haapakoski J, et al. (2001) Efficacy of a pneumococcal conjugate vaccine against acute otitis media. $\mathrm{N}$ Engl J Med 344: 403-409.

11. Fireman B, Black SB, Shinefield HR, Lee J, Lewis E, et al. (2003) Impact of the pneumococcal conjugate vaccine on otitis media. Pediatr Infect Dis J 22: 10-16.

12. Palmu AA, Verho J, Jokinen J, Karma P, Kilpi TM (2004) The seven-valent pneumococcal conjugate vaccine reduces tympanostomy tube placement in children. Pediatr Infect Dis J 23: 732-738. 
Citation: Ceyhan M, Ozsurekci Y, Gürler N, Oncel EK, Celik M, et al. (2013) Bacterial Etiology and Pneumococcal Serotypes in Turkish Children with Acute Otitis Media. J Vaccines Vaccin 5: 214. doi: 10.4172/2157-7560.1000214

13. Grall N, Hurmic O, Al Nakib M, Longo M, Poyart C, et al. (2011) Epidemiology of Streptococcus pneumoniae in France before introduction of the PCV-13 vaccine. Eur J Clin Microbiol Infect Dis 30: 1511-1519.

14. Leibovitz E, Serebro M, Givon-Lavi N, Greenberg D, Broides A, et al. (2009) Epidemiologic and microbiologic characteristics of culture-positive spontaneous otorrhea in children with acute otitis media. Pediatr Infect Dis J 28: 381-384.

15. McCaig LF, Hughes JM (1995) Trends in antimicrobial drug prescribing among office-based physicians in the United States. JAMA 273: 214-219.

16. Parra MM, Aguilar GM, Echaniz-Aviles G, Rionda RG, Estrada Mde L, et al. (2011) Bacterial etiology and serotypes of acute otitis media in Mexican children. Vaccine 29: 5544-5549.

17. Hotomi M, Togawa A, Kono M, Ikeda Y, Takei S, et al. (2013) PspA family distribution, antimicrobial resistance and serotype of Streptococcus pneumoniae isolated from upper respiratory tract infections in Japan. PLoS One 8: e58124.

18. Liu C, Xiong X, Xu W, Sun J, Wang L, et al. (2013) Serotypes and patterns of antibiotic resistance in strains causing invasive pneumococcal disease in children less than 5 years of age. PLoS One 8: e54254.

19. Bluestone CD, Stephenson JS, Martin LM (1992) Ten-year review of otitis media pathogens. Pediatr Infect Dis J 11: S7-11.

20. Block SL (1997) Causative pathogens, antibiotic resistance and therapeutic considerations in acute otitis media. Pediatr Infect Dis J 16: 449-456.

21. Arguedas A, Dagan R, Soley C, Loaiza C, Knudsen K, et al. (2003) Microbiology of otitis media in Costa Rican children, 1999 through 2001. Pediatr Infect Dis J 22: 1063-1068.

22. Li WC, Chiu NC, Hsu CH, Lee KS, Hwang HK, et al. (2001) Pathogens in the middle ear effusion of children with persistent otitis media: implications of drug resistance and complications. J Microbiol Immunol Infect 34: 190-194.

23. Guven M, Bulut Y, Sezer T, Aladag I, Eyibilen A, et al. (2006) Bacterial etiology of acute otitis media and clinical efficacy of amoxicillin-clavulanate versus azithromycin. Int J Pediatr Otorhinolaryngol 70: 915-923.
24. Oğuz F, Unüvar E, Süoğlu Y, Erdamar B, Dündar G, et al. (2003) Etiology of acute otitis media in childhood and evaluation of two different protocols of antibiotic therapy: 10 days cefaclor vs. 3 days azitromycin. Int J Pediatr Otorhinolaryngol 67: 43-51.

25. Alonso M, Marimon JM, Ercibengoa M, Perez-Yarza EG, Perez-Trallero E (2013) Dynamics of Streptococcus pneumoniae serotypes causing acute otitis media isolated from children with spontaneous middle-ear drainage over a 12year period (1999-2010) in a region of Northern Spain. PLoS One 8: e54333.

26. Reijtman V, Fossati S, Hernandez C, Sommerfleck P, Bernaldez P, et al. (2013) Serotype distribution of pneumococci isolated from pediatric patients with acute otitis media and invasive infections, and potential coverage of pneumococcal conjugated vaccine. Rev Argent Microbiol 45: 27-33.

27. Aguilar L, Alvarado O, Soley C, Abdelnour A, Dagan R, et al. (2009) Microbiology of the middle ear fluid in Costa Rican children between 2002 and 2007. Int J Pediatr Otorhinolaryngol 73: 1407-1411.

28. Zhou F, Kyaw MH, Shefer A, Winston CA, Nuorti JP (2007) Health care utilization for pneumonia in young children after routine pneumococcal conjugate vaccine use in the United States. Arch Pediatr Adolesc Med 161: 1162-1168.

29. Tsai CJ, Griffin MR, Nuorti JP, Grijalva CG (2008) Changing epidemiology of pneumococcal meningitis after the introduction of pneumococcal conjugate vaccine in the United States. Clin Infect Dis 46: 1664-1672.

30. Ma X, Zhao R, Ma Z, Yao K, Yu S, et al. (2013) Serotype distribution and antimicrobial resistance of Streptococcus pneumoniae isolates causing invasive diseases from Shenzhen Children's Hospital. PLoS One 8: e67507.

31. Bulut $Y$, Güven M, Otlu B, Yenişehirli G, Aladağ I, et al. (2007) Acute otitis media and respiratory viruses. Eur J Pediatr 166: 223-228.

32. Brook I, Gober AE (2000) Reliability of the microbiology of spontaneously draining acute otitis media in children. Pediatr Infect Dis J 19: 571-573.

33. Chen YJ, Hsieh YC, Huang YC, Chiu CH (2013) Clinical manifestations and microbiology of acute otitis media with spontaneous otorrhea in children. Microbiol Immunol Infect 46: 382-388. 